\title{
Asymptomatic Acute Ischemic Stroke After Primary Percutaneous Coronary Intervention in Patients With Acute Coronary Syndrome Might be Caused Mainly by Manipulating Catheters or Devices in the Ascending Aorta, Regardless of the Approach to the Coronary Artery
}

\author{
Motonobu Murai, MD; Hiroshi Hazui, MD*; Akira Sugie, MD*; Masaaki Hoshiga, MD; \\ Nobuyuki Negoro, MD; Hideyuki Muraoka, MD*; Hiroyuki Miyamoto, MD*; \\ Hitoshi Kobata, MD*; Hitoshi Fukumoto, MD*; Tadashi Ishihara, MD; \\ Hiroshi Morita, MD*; Toshiaki Hanafusa, MD
}

\begin{abstract}
Background Asymptomatic acute ischemic stroke (aAIS) following primary percutaneous coronary intervention (p-PCI) in patients with acute coronary syndrome (ACS) has not been studied in detail.

Methods and Results Of 75 patients who underwent p-PCI, 26 (34.7\%) developed aAIS as determined by diffusion-weighted magnetic resonance imaging (MRI). Including the approach to the coronary artery (via lower limb or right upper limb), 23 factors were compared between patients with $(n=26)$ and without $(n=49)$ aAIS. Age, hypertension, smoking, plasma glucose levels, Killip grade, right coronary artery (RCA) as culprit vessel, percutaneous coronary intervention (PCI) time, and the frequency of device insertion into the coronary artery differed in a statistically significant manner. However, multivariate analysis showed that the RCA (odds ratio 3.477) and the frequency of device insertion (1.375) were independent factors linked to the incidence of aAIS. Moreover, anterior or posterior location and left or right cerebral circulation of aAIS were equivalent in both approaches.

Conclusions Cranial MRI images following emergency PCI revealed that $34.7 \%$ of the patients with ACS had aAIS that might be caused by manipulating the catheter or devices in the ascending aorta, micro-air bubble embolism during injection, or micro-thrombus embolism derived from the ACS lesions during the PCI procedure. (Circ J 2008; 72: 51-55)
\end{abstract}

Key Words: Asymptomatic acute ischemic stroke; Diffusion weighted image; Primary percutaneous coronary intervention

$\mathbf{E}$ arly revascularization of the culprit vessels is the key to maintaining cardiac function in patients with acute coronary syndrome (ACS) $)_{1,2}$ and primary percutaneous coronary intervention (p-PCI) is one of the most effective ways of achieving this goal, 3 However, such intervention is also a recognized cause of the serious complication of symptomatic acute ischemic stroke (sAIS), although the incidence is rare $(0.11-0.38 \%)^{5-8}$ Portions of atherosclerotic plaque or thrombus, and air bubbles ${ }^{9,10}$ have been suggested causes of this complication and in particular, protruding aortic atheromas located distal to the innominate artery have been identified by transesophageal ultrasonography!1,12 Those findings indicate that approaching the coro-

(Received September 23, 2006; revised manuscript received August 20, 2007; accepted September 5, 2007)

1st Department of Internal Medicine, Osaka Medical College, *Department of Emergency Medicine, Osaka Mishima Emergency and Critical Care Center, Takatsuki, Japan

Mailing address: Motonobu Murai, MD, 1st Department of Internal Medicine, Osaka Medical College, 7-2 Daigaku-machi, Takatsuki 5698686, Japan. E-mail: in1288@ poh.osaka-med.ac.jp

All rights are reserved to the Japanese Circulation Society. For permissions, please e-mail: cj@j-circ.or.jp nary artery via the right upper limb could efficiently avoid this complication. However, differences in the incidence of sAIS after either the femoral artery or right upper limb approach to the coronary artery during catheter procedures have been examined in only 2 small retrospective studies of patients with symptomatic ischemic stroke! 13,14

Recent advances in magnetic resonance imaging (MRI), such as diffusion-weighted imaging (DWI), have detected small and hyperacute lesions of ischemic stroke, even in patients without clinical symptoms!5-20 The incidence of asymptomatic AIS (aAIS) after left ventriculography, ${ }^{18}$ coronary angiography, or elective percutaneous coronary intervention (PCI) ${ }^{19}$ as determined using DWI, has been reported; however, aAIS detected by MRI after p-PCI in patients with ACS has not been described, although sAIS follows PCI more often in the setting of urgency or emergency?

The present study examined the incidence and location of aAIS following p-PCI and related factors, including the effect of different approaches to the coronary artery. 

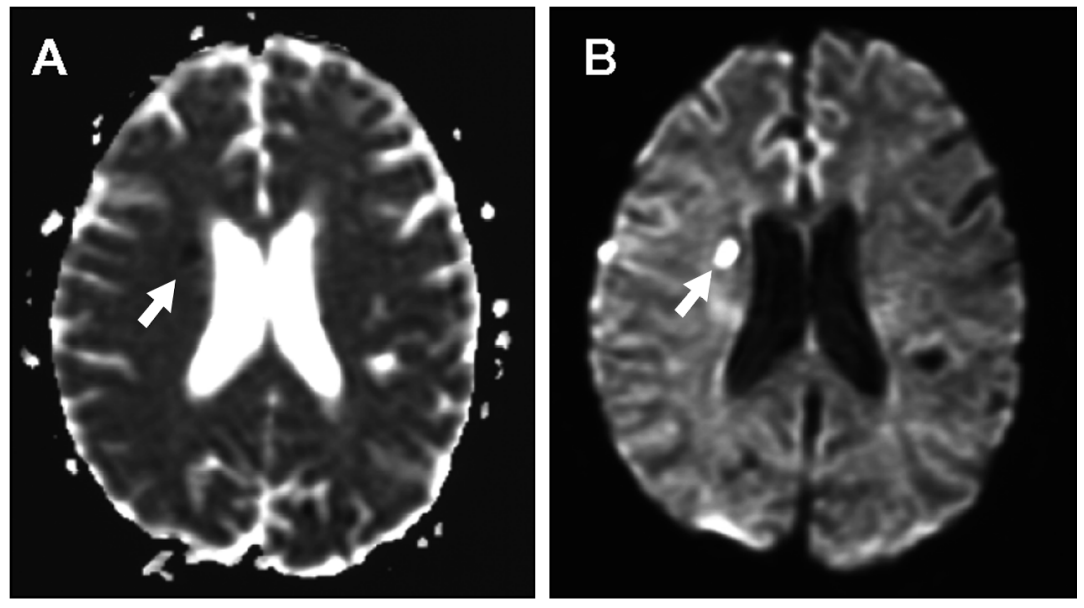

Fig 1. A 64-year-old male with hypertension and diabetes mellitus underwent primary percutaneous coronary intervention. Cranial magnetic resonance imaging performed 5 days later shows an acute ischemic stroke lesion of $5 \mathrm{~mm}$ in diameter located in the right corona radiate. Apparent diffusion coefficient (A) shows an area of low intensity and the diffusion-weighted image (B) shows an area of high intensity.

\section{Methods}

\section{Study Patients}

This single-center, prospective study included 75 consecutive patients with ACS and p-PCI (study group) and 15 consecutive patients with ACS but without p-PCI because of absence of ongoing ischemia (control group), excluding patients with prohibitions for MRI, out-of-hospital cardiac arrest or circulatory support such as intra-aortic counterpulsation or percutaneous cardiopulmonary support. The diagnosis of ACS was defined as typical chest oppression and ischemic changes on electrocardiograms. All participants were admitted to the Osaka Mishima Emergency and Critical Care Center between November, 2004 and December, 2005. The Human Research Committee of Osaka Mishima Emergency and Critical Care Center approved the study protocol.

\section{Catheter Procedure}

Four cardiologists randomly performed p-PCI immediately after admission. After oral administration of $162 \mathrm{mg}$ of aspirin, the first 49 consecutive patients underwent p-PCI through the right or left femoral artery (lower limb) using a 7Fr guiding catheter (Launcher Medtronic Inc, Minneapolis, $\mathrm{MN}$, USA) and the next 26 consecutive patients underwent the procedure through the right radial or brachial artery (upper limb) using a 6 Fr guiding catheter (Launcher Medtronic Inc). Only the cobalt-chromium alloy, coronary DRIVER stent (Medtronic Inc, Minneapolis, MN, USA) was implanted according to operator discretion because of its nonferromagnetic properties. After a sheath was inserted, $3,000 \mathrm{U}$ of unfractionated heparin was administered and another 5,000 $\mathrm{U}$ were added before the initiation of p-PCI.

\section{MRI Assessment}

All participants provided written informed consent to cranial MRI (ExcelART 1.5T, Toshiba, Japan), including FLAIR, DWI, T2*, and magnetic resonance angiography (MRA). The control and study groups underwent these procedures upon admission and within a mean of $3.0 \pm 1.0$ days after PCI, respectively. One or more physicians attended the patients during MRI. Lesions that generated high intensity with a low apparent diffusion coefficient value on DWI were considered to be aAIS 15

Patients in the study group were assigned to subgroups based on the presence or absence of aAIS. Cranial MRI images were interpreted by 2 strokologists who were unaware of the subgroup sequencing of the patients. The inter- and intra-observer reliability was excellent (kappa value ranging from 0.92 to 1.00) for detection of aAIS on cranial MRI.

\section{Study Protocol}

We initially compared the control and study groups for the incidence of aAIS and stenosis of $50 \%$ or more in the extracranial carotid artery on MRA, as well as the left ventricular ejection fraction (EF) using transthoracic echocardiography. Thereafter, we compared the following factors between the aAIS and non-aAIS groups: age, gender, coronary risk factors (smoking, hyperlipidemia, diabetes mellitus and hypertension), $\geq 50 \%$ stenosis in the extracranial carotid arteries on MRA, laboratory data on admission (mass creatine kinase isozyme MB (CKMB), total cholesterol, blood sugar and D-dimer values), maximum mass CKMB (measured over $3 \mathrm{~h}$ ), with or without multivessel disease ( $\geq 75 \%$ stenosis on angiography, except for culprit vessel), mean blood arterial pressure on admission, with or without Killip II or more, EF on transthoracic echocardiography and days from admission to measurement of EF, culprit vessel (right coronary artery (RCA) or not), time taken to complete PCI (PCI time), activated coagulation time (ACT) after PCI, difference in approach to the coronary artery (lower limb or right upper limb) and the frequency of insertion into the coronary artery of devices such as balloons, stent balloons or aspiration devices, operator experience with using cardiac catheters ( $\geq 10$ years or $<10$ years) with or without using aspiration devices. Moreover, the location (anterior or posterior cerebral circulation and left or right hemisphere) of the aAIS was also compared between the 2 approaches.

\section{Statistical Analysis}

The frequency and ratio (\%) of categorical data were determined and we calculated the means and standard deviation of continuous variables. Continuous variables were compared between the 2 groups using the Mann-Whitney U test. Demographic variables for categorical data were compared using the chi-square or Fischer exact test where appropriate. Statistically significant factors were examined using multivariate logistic regression. A value of $p<0.05$ was considered significant. All statistics were calculated using commercially available statistical software (SPSS II for Windows, Version 11.0; Chicago, IL, USA). 
Table 1 Characteristics of the Study Group

\begin{tabular}{lccc}
\hline \hline & $\begin{array}{c}\text { aAIS group } \\
(n=26)\end{array}$ & $\begin{array}{c}\text { Non-aAIS group } \\
(n=49)\end{array}$ & p value \\
\hline Age & $68.0 \pm 10.4$ & $61.8 \pm 11.2$ & 0.025 \\
Male & $18(69.2 \%)$ & $39(79.6 \%)$ & 0.474 \\
Smoking & $12(46.2 \%)$ & $34(69.4 \%)$ & 0.049 \\
Hyperlipidemia & $12(46.2 \%)$ & $26(53.1 \%)$ & 0.569 \\
Diabetes mellitus & $15(57.7 \%)$ & $18(36.7 \%)$ & 0.082 \\
Hypertension & $16(61.5 \%)$ & $18(36.7 \%)$ & 0.040 \\
Carotid stenosis $(\geq 50 \%)$ & $7(26.9 \%)$ & $6(12.2 \%)$ & 0.201 \\
\hline
\end{tabular}

aAIS, asymptomatic acute ischemic stroke.

Carotid stenosis was evaluated on magnetic resonance angiography.
Table 2 Laboratory Data on Admission

\begin{tabular}{lccc}
\hline \hline & $\begin{array}{c}\text { aAIS group } \\
(n=26)\end{array}$ & $\begin{array}{c}\text { Non-aAIS group } \\
(n=49)\end{array}$ & p value \\
\hline Mass CKMB $(\mathrm{ng} / \mathrm{ml})$ & $12.2 \pm 24.9$ & $22.1 \pm 57.7$ & 0.880 \\
TC $(\mathrm{mg} / \mathrm{dl})$ & $206 \pm 47.0$ & $218 \pm 36.1$ & 0.079 \\
Blood sugar $(\mathrm{mg} / \mathrm{dl})$ & $217 \pm 84.6$ & $187 \pm 83.2$ & 0.033 \\
D-dimer $(\mu \mathrm{g} / \mathrm{ml})$ & $1.6 \pm 1.3$ & $1.1 \pm 0.6$ & 0.134 \\
\hline
\end{tabular}

Mass CKMB, mass creatine kinase isozyme MB; TC, toal cholesterol. Other abbreviation see in Table 1.

Table 3 Severity of Acute Coronary Syndrome

\begin{tabular}{lccc}
\hline \hline & $\begin{array}{c}\text { aAIS group } \\
(n=26)\end{array}$ & $\begin{array}{c}\text { Non-aAIS group } \\
(n=49)\end{array}$ & $p$ value \\
\hline Max-mass CKMB $(\mathrm{ng} / \mathrm{ml})$ & $208.9 \pm 209.4$ & $242.8 \pm 206.0$ & 0.379 \\
Multi vessel disease & $11(42.3 \%)$ & $14(28.6 \%)$ & 0.230 \\
Mean blood pressure on admission $(\mathrm{mmHg})$ & $99 \pm 24$ & $97 \pm 20$ & 0.563 \\
Killip II or more on admission & $9(34.6 \%)$ & $6(17.2 \%)$ & 0.045 \\
Ejection fraction after admission $(\%)$ & $58 \pm 10$ & $56 \pm 7.7$ & 0.466 \\
\hline
\end{tabular}

Max-mass CKMB, maximum mass CKMB. Other abbreviations see in Tables 1,2.

Ejection fraction was measured by transthoracic ultrasoundsonography.

Table 4 Catheter Procedure

\begin{tabular}{lccc}
\hline \hline & $\begin{array}{c}\text { aAIS group } \\
(n=26)\end{array}$ & $\begin{array}{c}\text { Non-aAIS group } \\
(n=49)\end{array}$ & $p$ value \\
\hline Culprit vessel $(R C A)$ & $17(65.4 \%)$ & $16(32.7 \%)$ & 0.007 \\
PCI time (min) & $65.3 \pm 23.1$ & $56.6 \pm 26.5$ & 0.040 \\
ACT $($ s) & $358 \pm 97.8$ & $317 \pm 75.6$ & 0.087 \\
Approach from lower limb & $16(61.5 \%)$ & $31(63.3 \%)$ & 0.883 \\
Frequency of device insertion & $6.5 \pm 3.7$ & $4.7 \pm 2.3$ & 0.022 \\
Catheter experience $(n \geq 10$ years) & $9(34.6 \%)$ & $15(30.6 \%)$ & 0.724 \\
Aspiration device & $13(50 \%)$ & $22(44.9 \%)$ & 0.673 \\
\hline
\end{tabular}

$R C A$, right coronary artery; PCI, percutaneous coronary intervention; ACT, activated coagulation time. Other abbreviation see in Table 1.

\section{Results}

Cranial MRI was completely and safely accomplished in all patients. None of the control patients had aAIS, whereas 26 in the study group had aAIS (0\% vs $34.7 \%, \mathrm{p}=0.017$ ), although EF and the incidence of carotid stenosis were statistically equivalent in both groups (EF: $54 \pm 9.6 \%$ vs $57 \pm 8.6 \%$, $\mathrm{p}=0.287$; carotid stenosis: $17.3 \%$ vs $26.7 \%, \mathrm{p}=0.623$ ). None of the patients with aAIS had any clinical symptoms. All aAIS lesions were round in shape and their diameter was $10 \mathrm{~mm}$ or less on DWI (Fig 1). The aAIS and non-aAIS groups finally comprised 26 and 49 patients, respectively.

\section{Patient Characteristics (Table 1)}

Age, incidence of smoking and hypertension significantly differed between the aAIS and non-aAIS groups, whereas gender, incidence of hyperlipidemia and diabetes mellitus, and prevalence of extracranial carotid artery stenosis did not.

\section{Laboratory Data on Admission (Table 2)}

Plasma glucose levels significantly differed between the 2 groups but mass CKMB, total cholesterol and D-dimer values did not.
Table 5 Multivariate Analysis for Statistical Significant Factors

\begin{tabular}{lcc}
\hline \hline & \multicolumn{1}{c}{ OR $(95 \%$ CI $)$} & $p$ value \\
\hline Age & $1.017(0.961-1.077)$ & 0.560 \\
Hypertension & $2.681(0.773-9.300)$ & 0.120 \\
Smoking & $0.391(0.105-1.462)$ & 0.163 \\
Blood sugar value & $1.000(0.993-1.008)$ & 0.936 \\
Killip II or more on admission & $2.210(0.547-8.931)$ & 0.266 \\
Culprit vessel (RCA) & $3.477(1.008-11.988)$ & 0.048 \\
PCI time (min) & $0.999(0.971-1.027)$ & 0.939 \\
The frequency of device insertion & $1.375(1.041-1.817)$ & 0.025 \\
\hline
\end{tabular}

OR, odds ratio; CI, confidence intervaln. Other abbreviations see in Table 4.

\section{Severity of ACS (Table 3)}

The 2 groups differed significantly in Killip grade upon admission, but maximum mass CKMB values, presence or absence of multivessel disease, mean blood arterial pressure on admission and EF after admission did not. Days from admission to measuring of EF were also equivalent in both groups (aAIS vs non-aAIS: $2.9 \pm 2.3$ vs $2.7 \pm 1.9$ days, $\mathrm{p}=0.746$ ).

\section{Catheter Procedure (Table 4)}

The PCI time, RCA as the culprit vessel and the frequency of device insertion into the coronary artery significantly 
Table 6 Difference in Location of aAIS Between Approaches

\begin{tabular}{lccc}
\hline \hline & $\begin{array}{c}\text { Approach from lower limb } \\
(n=31)\end{array}$ & $\begin{array}{c}\text { Approach from right upper limb } \\
(n=55)\end{array}$ & p value \\
\hline Right hemisphere & $15(48 \%)$ & $28(51 \%)$ & 0.822 \\
Left hemisphere & $16(52 \%)$ & $27(49 \%)$ & 0.231 \\
Anterior cerebral circulation & $21(68 \%)$ & $30(55 \%)$ & \\
Posterior cerebral circulation & $10(32 \%)$ & $25(45 \%)$ & \\
\hline
\end{tabular}

Abbreviation see in Table 1.

differed between the groups, but ACT after p-PCI and the effect of the approach, operator experience with cardiac catheter (catheter experience), and use of aspiration devices (aspiration device) did not. In addition, because we used a single guiding catheter in all cases, the frequency of changing the guiding catheter did not differ between the 2 groups.

\section{Multivariate Analysis (Table 5)}

Age, incidence of hypertension and smoking, blood sugar level, Killip grade, RCA as culprit vessel, PCI time and the frequency of device insertion into the coronary artery were statistically re-evaluated using logistic regression. Only RCA as the culprit vessel and the frequency of device insertion into the coronary artery were indicated as independent factors related to the incidence of aAIS.

\section{Difference Between Approaches in the Location of aAIS (Table 6)}

Approaching from the lower or right upper limb resulted in 31 and 55 aAIS lesions, respectively. The location of the aAIS, such as anterior or posterior cerebral circulation and left or right hemisphere, was statistically equivalent for both approaches.

\section{Discussion}

\section{Concept That Catheter Manipulation Induces aAIS}

In the present study, aAIS after p-PCI in patients with ACS was frequently identified according to DWI. The size and shape of the lesion on the DWI suggested that embolic stroke was the cause of aAIS. An embolic source in ACS patients with p-PCI can be explained as follows: catheter complication, left ventricular thrombus and carotid arteryartery embolism. We found that ACS patients without p-PCI (control group) did not have aAIS lesions, although cardiac function represented by the EF was the same in both control and study groups. In addition, the severity of ACS, represented by maximum mass $\mathrm{CKMB}$ or the $\mathrm{EF}$, and the D-dimer value, which can indicate thrombus, did not significantly differ between the aAIS and non-aAIS group. These data suggest that left ventricular thrombus was probably not the embolic source. Moreover, we also evaluated the relationship between aAIS and the prevalence of extracranial carotid artery stenosis, which can cause aAIS by arteryto-artery embolism in patients with coronary artery disease, as well as within the general Japanese in population ${ }^{21}$ However, the number of patients with extracranial carotid artery stenosis did not statistically differ between the aAIS and non-aAIS groups. We thus determined that in the present study catheter manipulation during p-PCI caused aAIS.

\section{Timing of aAIS During p-PCI}

Reports from studies using transesophageal ultrasound suggest that atheromas in the aortic arch constitute an embolic source in patients with ischemic stroke or transient ischemic attack, ${ }^{11,12}$ which suggests that approaching the coronary artery from the right upper limb is safer because the catheter avoids passing through the aortic arch.

However, we compared the occurrence of aAIS between the approach via the lower or right upper limb and found no difference. On the other hand, we found that the independent factors related to aAIS following p-PCI were RCA as the culprit vessel and the frequency of device insertion into the coronary artery. These results suggest that aAIS mainly occurs either while manipulating the catheter or device at the bottom of the ascending aorta, which scratches the aortic wall ${ }^{22}$ when pulling a thrombus through the coronary artery using devices, or when pushing air bubbles out of the guiding catheters while using devices or contrast medium, 10 but not while moving the catheter to the ascending aorta, as reported by Dawson et al!3 The reason why the RCA lesion is significantly related to the incidence of aAIS is unknown. We suggest that 1 possibility is that the right coronary catheter must be rotated to engage the RCA and the likelihood of scratching the aorta is high as compared with the left coronary catheter, which will naturally seek the ostium of the left coronary artery. Kosmorsky et al showed in a study of 10 patients with symptomatic stroke that those who underwent left heart catheter examination via an approach from the right upper limb were susceptible to vertebrobasilar stroke.$^{14}$ However, we did not find any significant differences in the distribution of aAIS between the 2 approaches, which supports the notion that aAIS is mainly caused by manipulating catheters or devices at the bottom of the ascending aorta. In addition, there was no difference in the operator's experience of using cardiac catheters.

\section{Comparison With Elective Catheter Procedure}

BÜsing et al identified silent brain ischemia in $11 \%$ of patients undergoing elective coronary angiography and in $23 \%$ of those having elective PCI ${ }^{19}$ The incidence of aAIS in our study was likely to be higher than in that study. Moreover, Lecker et al revealed that 12 of 16 patients developed acute symptomatic stroke after emergency catheterization? Thus, patients who undergo emergency catheterization for events such as ACS might be predisposed to embolism because of high coagulability. Another possible mechanism of the high frequency of aAIS in ACS patients is distal emboli of fragile plaque from the coronary ostial lesion, in particular when thrombus aspiration devices are used. However, we could not find a statistical difference between the aAIS and non-aAIS groups in the frequency of using thrombus aspiration devices. Further investigations are required in similar institutions to compare the incidence of aAIS after primary PCI with that after elective PCI.

Clinical Significance of aAIS

Interestingly, silent brain ischemia has been associated 
with dementia or depression. Fujikawa et al identified silent brain ischemia on MRI in $51.4 \%$ of patients with pre senile-onset, pre-senile depression, in $65.9 \%$ of those with pre senile-onset senile depression and in $93.7 \%$ of those with senile-onset depression. ${ }^{23}$ In addition, Vermeer et al concluded from a mean 3.6-year follow-up of 1,015 participants that the presence of silent brain ischemia at baseline more than doubled the risk of dementia 24 Although we cannot state that the silent brain ischemia detected in their studies is equivalent to the aAIS observed in our study, the relationship between aAIS after PCI and dementia or depression should be investigated.

\section{Study Limitations}

One limitation of the present study is that cranial MRI was not performed before p-PCI for ethical reasons. However, aAIS did not appear on preprocedural cranial MRI according to Omran et $\mathrm{al}{ }^{18}{ }^{1}$ BÜsing et al ${ }^{19}$ and Restrepo et $\mathrm{al}^{20}$ as in our control group. Thus, it seems rare to incidentally find aAIS lesions with high intensity and a low apparent diffusion coefficient value on DWI. Hence, we assumed that aAIS did not exist before p-PCI in our study group. Another limitation is that this study was a single center trial comprised of relatively few patients and the number of those with aAIS was small. Therefore, the results of the present study should be interpreted with caution.

\section{Conclusions}

Cranial MRI imaging following emergency PCI revealed that $34.7 \%$ of the patients with ACS had aAIS that might be caused by manipulating either the catheter or devices in the ascending aorta, micro-air-bubble embolism during injection, or micro-thrombus embolism derived from the ACS lesions during the PCI procedure. Different approaches did not affect the location or incidence of aAIS.

\section{References}

1. Brodie BR, Stuckey TD, Muncy DB, Hansen CJ, Wall TC, Pulsipher $\mathrm{M}$, et al. Importance of time-to-reperfusion in patients with acute myocardial infarction with and without cardiogenic shock treated with primary percutaneous coronary intervention. Am Heart J 2003; 145: $708-715$.

2. Watanabe K, Nagao K, Watanabe I, Kanmatsuse K. Relationship between the door-to-TIMI-3 flow time and the infarct size in patients suffering from acute myocardial infarction. Circ $J$ 2004; 68: $280-$ 285.

3. Degeare VS, Dangas G, Stone GW, Grines CL. Interventional procedures in acute myocardial infarction. Am Heart J 2001; 141: 15-24.

4. Aversano T, Aversano LT, Passamani E, Knatterud GL, Terrin ML, Williams DO, et al. Thrombolytic therapy versus primary percutaneous coronary intervention for myocardial infarction in patients presenting to hospitals without on-site cardiac surgery: A randomized controlled trial. JAMA 2002; 287: 1943-1951.
5. Segal AZ, Abernethy WB, Palacios IF, BeLue R, Rordorf G. Stroke as a complication of cardiac catheterization: Risk factors and clinical features. Neurology 2001; 56: 975-977.

6. Fuchs S, Stabile E, Kinnaird TD, Mintz GS, Gruberg L, Canos DA, et al. Stroke complicating percutaneous coronary interventions; incidence, predictors, and prognostic implications. Circulation 2002; 106: $86-91$.

7. Leker RR, Pollak A, Abramsky O, Ben-Hur T. Abundance of left hemispheric embolic strokes complicating coronary angioplasty and PTCA. J Neurol Neurosurg Psychiatry 1999; 66: 116-117.

8. Dukkipati S, O'Neill WW, Harjai KJ, Sanders WP, Deo D, Boura JA, et al. Characteristics of cerebrovascular accidents after percutaneous coronary interventions. J Am Coll Cardiol 2004; 43: 1161-1167.

9. Wijman CAC, Kase CS, Jacobs AK, Whitehead RE. Cerebral air embolism as a cause of stroke during cardiac catheterization. Neurology 1998; 51: 318-319.

10. Stygall J, Kong R, Walker JM, Hardman SMC, Harrison MJG, Newman SP. Cerebral microembolism detected by transcranial doppler during cardiac procedures. Stroke 2000; 31: 2508-2520.

11. Tunick PA, Perez JL, Kronzon I. Protruding atheromas in the thoracic aorta and systemic embolization. Ann Intern Med 1991; 115: $423-427$.

12. Jones EF, Kalman JM, Calafiore P, Tonkin AM, Donnan GA. Proximal aortic atheroma; an independent risk factor for cerebral ischemia. Stroke 1995; 26: 218-224.

13. Dawson DM, Fischer EG. Neurologic complications of cardiac catheterization. Neurology 1977; 27: 496-497.

14. Kosmorsky G, Hanson MR, Tomsak RL. Neuro-ophthalmologic complications of cardiac catheterization. Neurology 1988; 38: 483-485.

15. Mosely ME, Kucharczyk J, Mintorovitch J, Cohen Y, Kurhanewicz J, Derugin N, et al. Diffusion-weighted MR imaging of acute stroke: Correlation with T2-weighted magnetic susceptibility-enhanced MR imaging in cats. Am J Neuroradiol 1990; 11: 423-429.

16. Warach S, Gaa J, Siewert B, Wielopolski P, Edelman RR. Acute human stroke studied by whole brain echo planar diffusion-weighed magnetic resonance imaging. Ann Neurol 1995; 37: 231-241.

17. Schaefer PW, Grant PE, Gonzalez G. Diffusion-weighted MR imaging of the brain. Radiology 2000; 217: 331-345.

18. Omran H, Schmidt H, Hackenbroch M, Clllien S, Bernhardt P, von der Recke G, et al. Silent and apparent cerebral embolism after retrograde catheterization of the aortic valve in valvular stenosis: A prospective, randomised study. Lancet 2003; 361: 1241-1246.

19. BÜsing KA, FlÜchter CSSS, SÜselbeck T, Hasse KK, Neff W, Hirsch JG, et al. Cerebral infarction: Incidence and risk factors after diagnostic and interventional cardiac catheterization-prospective evaluation at diffusion-weighted MR imaging. Radiology 2005; 235: $177-183$.

20. Restrepo L, Wityk RJ, Grega MA, Borowicz L, Barker PB, Jacobs MA, et al. Diffusion- and perfusion- weighted magnetic resonance imaging of the brain before and after coronary artery bypass grafting surgery. Stroke 2002; 33: 2909-2915.

21. Tanimoto T, Ikari Y, Tanabe K, Yachi S, Nakajima H, Nakayama T. Prevalence of carotid artery stenosis in patient with coronary artery disease in Japanese population. Stroke 2005; 36: 2094-2098.

22. Galbreath C, Salgado ED, Furlan AJ, Hollman J. Central nervous system: Complications of percutaneous transluminal coronary angiography. Stroke 1986; 17: 616-619.

23. Fujikawa T, Yamawaki S, Touhouda Y. Incidence of silent cerebral infarction in patients with major depression. Stroke 1993; 24: 1631 1634.

24. Vermeer SE, Prins ND, den Heijer T, Hofman A, Koudstaal J, Breteler MMB, et al. Silent brain infarcts and the risk of dementia and cognitive decline. N Engl J Med 2003; 348: 1215-1222. 